\title{
Expression of NF-kB and Occludin in Intestinal Mucosal Barrier During Severe Acute Pancreatitis and Its Clinical Significance
}

\author{
Qingyun Zhu ${ }^{1}$, Shigang Yang ${ }^{2}$, Xinting Pan ${ }^{1}{ }^{*}$, Chonggao Yin ${ }^{1}$, Shijie Liu ${ }^{1}$, Fuguo Liu ${ }^{1}$, Ying Sun ${ }^{1}$, \\ Huimin Wang ${ }^{1}$, Yajun Jing ${ }^{1}$ \\ ${ }^{1}$ Department of Intensive Care Unit, The Affiliated Hospital of Qingdao University, Qingdao, China \\ ${ }^{2}$ Department of Gastroenterology, The Qingzhou Huanglou Hospital, Qingzhou, China
}

Email address:

0536pxt@163.com (Xinting Pan)

${ }^{*}$ Corresponding author

\section{To cite this article:}

Qingyun Zhu, Shigang Yang, Xinting Pan, Chonggao Yin, Shijie Liu, Fuguo Liu, Ying Sun, Huimin Wang, Yajun Jing. Expression of NF-kB and Occludin in Intestinal Mucosal Barrier During Severe Acute Pancreatitis and Its Clinical Significance. American Journal of Internal Medicine. Vol. 5, No. 5, 2017, pp. 70-73. doi: 10.11648/j.ajim.20170505.11

Received: July 5, 2017; Accepted: July 14, 2017; Published: August 7, 2017

\begin{abstract}
Objective to investigate the expression of NF- $\kappa \mathrm{B}$ and occludin in intestinal mucosal barrier during severe acute pancreatitis (SAP) and its clinical significance. Method Twenty-four Wistar rats were randomly divided into a normal control group, an SAP group, and a PDTC group. The rats were sacrificed at $24 \mathrm{~h}$ after modeling, and general appearance of the abdominal cavity, pathological changes of the intestinal mucosa, changes in intestinal mucosal permeability were observed, serum inflammatory factor IL-1 was measured, and expression of tight junction protein occludin in the intestinal epithelial cells were determined by immunohistochemistry. Results Rats of the SAP group showed obvious inflammatory reaction in the abdominal cavity, manifested by enlargement of intestinal tract, intestinal edema, and increased permeability of intestinal barrier, whereas NF- $\mathrm{B}$ inhibitor alleviated intestinal damage and intestinal mucosa edema, upregulated intestinal epithelial tight junction protein occludin, decreased intestinal permeability, and lowered the level of inflammatory factors. Conclusion Impairment of intestinal barrier function during SAP may be associated with increased NF- $\kappa \mathrm{B}$ and decreased intestinal tight junction protein occludin; inhibiting NF-kB may alleviate intestinal mucosal barrier dysfunction during SAP.
\end{abstract}

Keywords: Severe Acute Pancreatitis, Intestinal Mucosal Barrier, Nf-Kb, Inflammatory Factor

\section{Introduction}

Severe acute pancreatitis (SAP) is a common severe clinical situation. This disease is manifested by structural and functional abnormalities of the intestinal epithelial tight junction and leads to increased intestinal mucosal permeability, increased translocation of intestinal bacteria and toxins, and secondary systemic infection and inflammatory response, which makes SAP a major cause of sepsis. Studies have shown that the major causes of intestinal mucosal barrier dysfunction, increased intestinal permeability, and intestine-derived infection are several cytokines and inflammatory mediators. NF- $\kappa \mathrm{B}$ was the intracellular signal transducer of multiple inflammatory cytokines, and play a key regulatory role in immune response and inflammation. In this study, we constructed a rat model of SAP, and observed the effect of specific $\mathrm{NF}-\kappa \mathrm{B}$ blocker on intestinal mucosal barrier function. Expression of tight junction protein occludin was also determined to clarify the mechanism how NF- $\mathrm{B}$ affect intestinal mucosal barrier impairment in SAP rats.

\section{Material and Methods}

\subsection{Animals, Agents, and Grouping}

Healthy male Wistar rats, weighing 250-340g, were provided by the Animal center of the Academy of Military Medical Science. Sodium taurocholate, NF-кB inhibitor 
pyrrolidine dithiocarbamate (PDTC), FITC-labeled dextran 4000 (FD 4) were purchased from Sigma; ELISA kit, anti-occludin goat polyclonal antibody ( $\mathrm{IgG}$ ) was purchased from Santa Cruz Biotechnology; immunohistochemistry kit, DAB staining kit, and anti-off slides were purchased from Fuzhou Maixin biotechnology.

24 Wistar rats were randomly divided into a normal control group, a SAP group, and a NF- $\mathrm{B}$ inhibitor (PDTC) group. The rats were fasted for $12 \mathrm{~h}$ before surgery, but allowed free access to water. The rats were anesthetized by intraperitoneal injection with $2.5 \%$ sodium pentobarbital at $1 \mathrm{ml} / \mathrm{kg}$. After routine disinfection, the belly was cut open right in the middle, and the bile duct was temporarily clamped with an arterial clip at the end of hepatic portal vein, a \#5 needle was reversely inserted into the pancreaticobiliary duct, which was then temporarily clamped with another arterial clip near the opening to the dodecadactylon. 5\% sodium taurocholate $(1.5 \mathrm{ml} / \mathrm{kg})$ was injected at $0.1 \mathrm{ml} / \mathrm{min}$, and the arterial clips were removed $10 \mathrm{~min}$ later, then the belly was sealed layer by layer. For the normal control group, the pancreas was flipped, and the PDTC group received $40 \mathrm{ml} / \mathrm{kg}$ PDTC solution via the tail vein $2 \mathrm{~h}$ after modeling. All groups were fasted but allowed free access to water after surgery. And normal saline was injected subcutaneously at $40 \mathrm{ml} / \mathrm{kg}$ to supplement for loss of body fluids. The rats were sacrificed $24 \mathrm{~h}$ after modeling and tissue samples were collected for analysis.

\subsection{Methods}

\subsubsection{Changes in General Appearance}

Intraperitoneal inflammation, and dilation, edema, and congestion of intestinal tract were observed.

\subsubsection{Intestinal Mucosal Pathology}

$10 \mathrm{~cm}$ of ileum was taken, fixed with $10 \%$ formaldehyde, and stained with Hematoxylin-eosin (HE). Then the slices were observed under a $400 \mathrm{X}$ light microscope. Mucosal injury score: 0 for normal mucosa; 1 for increased villus apical subepithelial space; 2 for moderate detachment of upper layer of epithelium from lamina propria mucosae; 3 for severe detachment with damage at the top of the villi; 4 for damaged villi and exposure of lamina propria mucosae vessles; 5 for destruction of lamina propria mucosae, bleeding, and ulcers. 10 visual field were selected for each segment, and the total score was calculated.

\subsubsection{Permeability of Isolated Intestinal Mucosa}

$10 \mathrm{~cm}$ of ileum was taken and was turned inside out, $0.6 \mathrm{ml}$ KHBB buffer was injected, and the two sides of the ileum was sealed. The segment was incubated in KHBB buffer that contained $500 \mu \mathrm{g} / \mathrm{mL}$ FD 4 at $37^{\circ} \mathrm{C}$ for $30 \mathrm{~min}$, then the KHBB buffer inside the segment was taken out to determine FD 4 concentration and to calculate permeability of the intestinal mucosa for FD 4, using the following equation: $\mathrm{C}=$ FD 4 (outside) $* 0.6 / \mathrm{FD} 4$ (inside) $* 30 \mathrm{~min} * \pi * I * \mathrm{~d}$, wherein, C represents permeability of the intestine for FD $4, I$ represent length of the intestine segment, and $d$ represents its diameter.

\subsubsection{Serum Inflammatory Factors}

Blood was collected by removing the eyeball to determine serum TNF- $\alpha$ and IL-1 concentrations.

\subsubsection{Detection of Occludin Expression by SP Immunohistochemistry}

Paraffin embedded sections were dewaxed, incubated in $\mathrm{H}_{2} \mathrm{O}_{2}$, underwent antigen repair, and blocked with serum. Then the slices were incubated with goat anti-rat multiclonal antibody $(1: 100)$ at $4{ }^{\circ} \mathrm{C}$ overnight, followed by incubation with biotinylated rabbit anti-goat $\mathrm{IgG}$ at $37^{\circ} \mathrm{C}$ for $30 \mathrm{~min}$, then a drop of appropriately diluted horseradish labeled streptavidin solution was added. The image was revealed using the DAB method, time of exposure was optimized by observing under a light microscope. The slices were further stained with hematoxylin, dehydrated, and mounted with resin. For blank control, PBS was used in place of primary antibody.

\subsection{Statistical Analysis}

Statistical analysis was performed using the SPSS 13.0 software package. Comparison between groups was performed using independent sample t-test, and $\mathrm{P}<0.05$ was considered statistically significant.

\section{Results}

\subsection{Changes in General Appearance of the Abdominal Cavity}

No significant signs of inflammation were observed with naked eye in abdominal cavity of the control group, i.e., no edema or bleeding. Rats of the SAP and PDTC group showed ascites in the abdominal cavity, with the symptoms being more severe in the SAP group.

\subsection{Intestinal Mucosal Pathology}

No damage to the intestinal mucosa was observed in the control group, while intestinal mucosal destruction was observed in the SAP and PDTC group. Inflammatory cell infiltration in the intestinal mucosa, interstitial edema, and irregular villus, mucosal erosion and necrosis, inflammatory exudate, and shredded intestinal epithelium were observed in the SAP group. Intestinal inflammatory cell infiltration, edema of the intestinal wall, the subcutaneous space expanding were also observed in the PDTC group, but milder than the SAP group (Figure 1). Pathological score of the control, SAP, and PDTC groups were $3.62 \pm 1.35,21.27 \pm$ 3.71 and $33.76 \pm 4.18$ respectively $(\mathrm{P}<0.05)$.

\subsection{Permeability of Isolated Intestinal Mucosa}

Permeability value of the control, SAP, and PDTC groups were $21.51 \pm 5.67,187.92 \pm 26.37$ and $328.49 \pm$ $34.81 \mathrm{nl} / \mathrm{min} \cdot \mathrm{cm}$ respectively, the values of both the SAP and PDTC groups increased compared to the control group 
$(\mathrm{P}<0.05)$, and the increase was to a higher extent in the SAP group.

\subsection{Serum Cytokines}

TNF- $\alpha$ levels of the control group, PDTC group and SAP group were $145.75 \pm 37.36,1683.56 \pm 79.81$, and $2896.32 \pm$ $98.78 \mathrm{pg} / \mathrm{mL}$ respectively, and IL-1 concentrations were $53.26 \pm 10.28,1756.32 \pm 59.65,2892.76 \pm 87.68 \mathrm{pg} / \mathrm{mL}$. Both TNF- $\alpha$ and IL-1 levels increased in the SAP and PDTC groups compared to the control group $(\mathrm{P}<0.05)$, and the increase was larger in the SAP group than the PDTC group $(\mathrm{P}<0.05)$.

\subsection{Detection of Occludin Expression by Immunohistochemistry}

Occludin of the control group was distributed along the intestinal epithelial cells, as indicated by the deeply stained brown line; localization of occludin in the PDTC group showed similar pattern to the control group, but the signal was weaker; while occludin-positive cells significantly decreased in the SAP group (Figure 2). IHS demonstrated occludin stain scores of $15.63 \pm 2.98,6.35 \pm 1.45$, and $3.08 \pm 1.21$ for the control group, the PDTC group, and SAP group respectively. The difference among the groups was statistically significant $(\mathrm{P}<0.05)$.

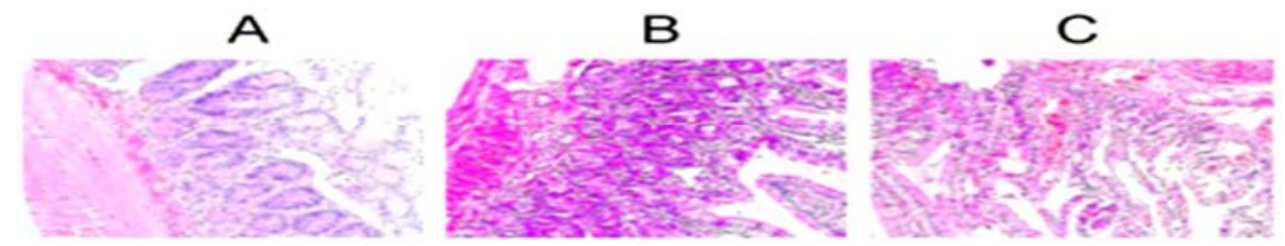

Figure 1. Pathological changes of the intestinal mucosa (H-E stain, $\times 100)$. A: Control group; B: PDTC group; C: SAP group.
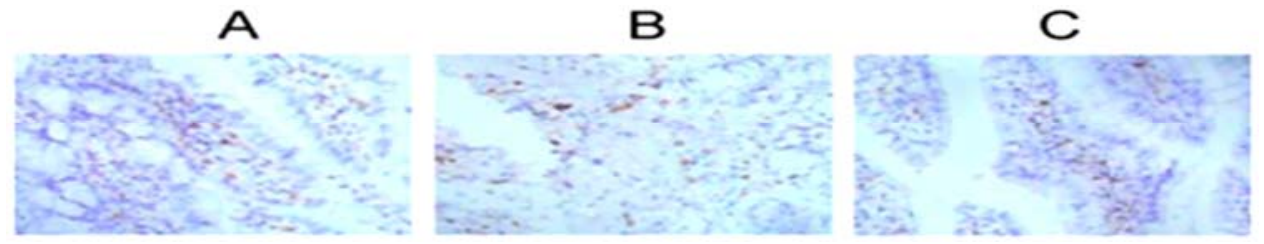

Figure 2. Immunohistochemistry staining of occludin (×100). A: Control group; B: PDTC group; C: SAP group.

\section{Discussion}

SAP is a common severe situation of the abdomen, despite great progresses in diagnosis, prevention and treatment of this disease, SAP remains a great threat to human lives (mortality being up to $10 \% \sim 25 \%$ ). [1, 2, 3] In the course of $\mathrm{SAP}$, the intestinal mucosa may undergo various structural and functional changes, which leads to intestinal mucosal barrier dysfunction. Studies have shown that translocation of bacteria through the intestinal wall represents the major cause of pancreatic and peripancreatic infection and sepsis, as well as systemic inflammatory response syndrome and multiple organ dysfunction, so it can't be overemphasized the importance of maintaining intestinal mucosal barrier integrity and preventing translocation of bacteria and endotoxins. [4, 5, 6, 7, 8] Epithelial cells and intercellular adhesion are the fundamental components of the intestinal mucosal barrier, and the latter includes tight junction, adhesion junction, gap junction, etc. The junction proteins between epithelial cells mainly include occludin, claudin, ZO-1, and so on, whereas occludin is one of the major functional components of tight junction between cells. [9, 10, 11, 12] Expression of this protein is regulated by multiple layers, and due to its vital role in maintaining intestinal mucosa barrier, its functional status is involved in the occurrence and progression of various diseases. Regulation of tight junction proteins is currently not very clear, whether this protein is also regulated by other major pathways requires further investigation.
In this study, levels of the inflammatory factors TNF- $\alpha$ and IL-1 significantly increased in the SAP group, which was accompanied by obvious inflammatory cell infiltration, edema of intestinal wall, and increased space below the intestinal villus. In addition, permeability test showed increased intestinal mucosal permeability, and expression of occludin in the intestinal epithelium significantly decreased. PDTC is an antioxidant, and recent studies have demonstrated that PDTC may inhibit activation of NF- $\kappa$ B by blocking degradation of I- $\kappa \mathrm{B}$. In this study, after inhibiting NF- $\kappa$ B with PDTC, serum TNF- $\alpha$ and IL-1 of the PDTC group significantly decreased compared to the untreated SAP rats, ileum damage significantly reduced, intestinal epithelium permeability significantly decreased, and occludin expression in intestinal epithelium significantly increased. These results suggest that NF- $\kappa \mathrm{B}$ may downregulate occludin expression, and thus aggravate dysfunction of intestinal mucosal barrier.

Toll-like receptor 4 (TLR 4) / NF- $\mathrm{KB} /$ cytokines (CKs) signaling pathway has been proposed as the key initiator involved in various diseases. [13, 14, 15, 16] TLR 4 recognizes the pathogen-associated molecular pattern and transduce the inflammatory response signal into the cell. Among the downstream effectors, NF- $\mathrm{BB}$ stands as a hub among the transcription factors, which normally exists in an inactive form with its inhibitory unit I- $\kappa B$. In case of SAP, endotoxins such as LPS enters into the blood circulation and get recognized by TLR 4 on cell surface, which ultimately leads to translocation of NF- $\mathrm{KB}$ from the cytoplasm into the 
nucleus, where NF- $\mathrm{BB}$ induces transcription of various inflammatory factors. Excessive production of inflammatory factors leads to systemic inflammatory response syndrome, sepsis, and multiple organ failure. It requires further investigation whether the TLR $4 / \mathrm{NF}-\kappa \mathrm{B} / \mathrm{CKs}$ signaling pathway exerts its impact on intestinal mucosal barrier function by regulating occludin expression.

In this study, PDTC inhibits the TLR $4 / \mathrm{NF}-\kappa \mathrm{B} / \mathrm{CKs}$ signaling pathway by selectively inhibiting $\mathrm{NF}-\kappa \mathrm{B}$, and significantly improves intestinal mucosal barrier function, reduces translocation of bacteria and endotoxin, and ultimately reduces systemic inflammatory response and the incidence of sepsis. Previous studies have proved that NF- $\mathrm{BB}$ signaling has a destructive effect on intestinal mucosal barrier function, but the mechanism has not been clarified. In this study, we examined occludin expression in the control group, the SAP group, and the PDTC group, and the results suggested that the intestinal mucosal barrier dysfunction in SAP may be due to activation of the TLR $4 / \mathrm{NF}-\kappa \mathrm{B} / \mathrm{CKs}$ signaling pathway and consequently decreased occludin expression.

In summary, increased NF- $\mathrm{B}$ expression in intestinal mucosa of SAP rats is of vital significance in the occurrence and progression of intestinal mucosal barrier dysfunction. By inhibiting NF- $\kappa \mathrm{B}$ activity with PDTC, structural and functional status of occludin, a major component of intestinal mucosal tight junction, can be improved. Our results provide a practical target for prevention and treatment of intestinal mucosal barrier dysfunction secondary to SAP.

\section{References}

[1] Jeon TJ, Park JY. Clinical significance of the neutrophil-lymphocyte ratio as an early predictive marker for adverse outcomes in patients with acute pancreatitis. World $\mathrm{J}$ Gastroenterol, 2017, 7; 23 (21): 3883-3889.

[2] Huang W, Windsor JA. Fulminant or Early Severe Acute Pancreatitis Is Over-looked by Classifications of Severity. Crit Care Med, 2017, 45 (7): e 744-e 745.

[3] Gomatos IP, Halloran CM, Ghaneh P, et al. Outcomes From Minimal Access Retroperitoneal and Open Pancreatic Necrosectomy in 394 Patients With Necrotizing Pancreatitis. Ann Surg, 2016, 263 (5): 992-1001.

[4] Deng W, Abliz A, Xu S, et al. Severity of pancreatitisassociated intestinal mucosal barrier injury is reduced following treatment with the NADPH oxidase inhibitor apocynin. Mol Med Rep, 2016, 14 (4): 3525-3534.
[5] Ouyang J, Zhang ZH, Zhou YX, et al. Up-regulation of Tight-Junction Proteins by p 38 Mitogen-Activated Protein Kinase/p 53 Inhibition Leads to a Reduction of Injury to the Intestinal Mucosal Barrier in Severe Acute Pancreatitis. Pancreas, 2016, 45 (8): 1136-1144.

[6] Yao P, Cui M, Li Y, et al. Effects of rhubarb on intestinal flora and toll-like receptors of intestinal mucosa in rats with severeacute pancreatitis. Pancreas, 2015, 44 (5): 799-804.

[7] $\mathrm{Xu} \mathrm{GF,} \mathrm{Guo} \mathrm{M,} \mathrm{Tian} \mathrm{ZQ,} \mathrm{et} \mathrm{al.} \mathrm{Increased} \mathrm{of} \mathrm{serum}$ high-mobility group box chromosomal protein 1 correlated with intestinalmucosal barrier injury in patients with severe acute pancreatitis. World J Emerg Surg, 2014, 17 (9): 61.

[8] Zhang XP, Jiang J, Yu YP, et al. Effect of Danshen on apoptosis and NF- $\kappa B$ protein expression of the intestinal mucosa of rats with severe acute pancreatitis or obstructive jaundice. Hepatobiliary Pancreat Dis Int, 2010, 9 (5): 537-546.

[9] Wang L, Cui YL, Zhang Z, et al. Rhubarb Monomers Protect Intestinal Mucosal Barrier in Sepsis via Junction Proteins. Chin Med J (Engl), 2017, 130 (10): 1218 -1225.

[10] Kong W, Huang C, Tang Y, et al. Effect of Bacillus subtilis on Aeromonas hydrophila-induced intestinal mucosal barrier function damage and inflammation in grass carp (Ctenopharyngodon idella). Sci Rep, 2017, 7 (1): 1588.

[11] Watari A, Sakamoto Y, Hisaie K, et al. Rebeccamycin Attenuates TNF- $\alpha$ - Induced Intestinal Epithelial Barrier Dysfunction by Inhibiting Myosin Light Chain Kinase Production. Cell Physiol Biochem, 2017; 41 (5): 1924-1934.

[12] Li W, Sun K, Ji Y, et al. Glycine Regulates Expression and Distribution of Claudin-7 and ZO-3 Proteins in Intestinal Porcine Epithelial Cells. J Nutr, 2016, 146 (5): 964-969.

[13] Yuan Y, Han Q, Li S, et al. Wnt 2 b attenuates HSCs activation and liver fibrosis through negative regulating TLR 4 signaling. Sci Rep, 2017, 7 (1): 3952.

[14] Irene Guijarro-Muñoz, Marta Compte, Ana Álvarez-Cienfuegos, et al. Lipopolysaccharide Activates Toll-like Receptor 4 (TLR 4)-mediated NF- $\kappa B$ Signaling Pathway and Proinflammatory Response in Human Pericytes J Biol Chem, 2014, 289 (4): 2457-2468.

[15] Serrero M, Planès R, Bahraoui E. PKC- $\delta$ isoform plays a crucial role in Tat-TLR 4 signalling pathway to activate NF- $\kappa B$ and CXCL 8 production. Sci Rep, 2017, 7 (1): 2384.

[16] Nyati KK, Masuda K, Zaman MM, et al. TLR 4-induced $N F-\kappa B$ and MAPK signaling regulate the IL-6 mRNA stabilizing protein Arid 5a. Nucleic Acids Res, 2017, 45 (5): 2687-2703. 